\title{
Clinical and whole-exome sequencing findings in Individuals from Yunnan Province with Familial Exudative Vitreoretinopathy
}

\author{
Zhen Zhang ( $\sim$ 2218458245@qq.com ) \\ Kunming Medical University \\ Yi-shuang Xiao \\ Kunming Medical University \\ Hong-chao Jiang \\ Kunming Medical University \\ Ru Sheng \\ Kunming University of Science and Technology \\ Li Tang \\ Kunming Medical University \\ XIAO-Hong Yang \\ Kunming Medical University \\ Huai-yu Gu \\ Sun Yat-Sen University \\ Wen-Ji He \\ Kunming Medical University \\ Jing Ma \\ Kunming Medical University
}

\section{Research}

Keywords: familial exudative vitreoretinopathy, whole-exome sequencing, genetic forms

Posted Date: January 15th, 2020

DOl: https://doi.org/10.21203/rs.2.20941/v1

License: (c) (i) This work is licensed under a Creative Commons Attribution 4.0 International License. Read Full License 


\section{Abstract}

Background : Familial exudative vitreoretinopathy (FEVR) is a rare inherited retinal disorder characterized by the failure of peripheral retinal vascularization at birth. FEVR can cause further pathological changes, such as neovascularization, exudation, haemorrhage, and retinal detachment, in turn. The molecular diagnosis enables a deeper understanding of this disease, so the differentiation of FEVR is important for the accuracy of genetic counselling. However, to date, only six genes have been identified as being responsible for this disease.

Methods : Without a known FEVR gene mutation, six families were enrolled in our study between 2016 and 2017 from the clinical practices of ophthalmologists. The referring physician made a diagnosis for FEVR probands, and clinical data and DNA were collected from each participant. Whole-exome sequencing was used to detect the mutations present in the probands. The raw reads were obtained from Illumina Miseq. Then, an in-house bioinformatics pipeline was performed to detect both single nucleotide variants (SNVs) and small insertions/deletions (InDels). The pathogenic mutations were identified with stringent criteria and were further confirmed by conventional methods and cosegregation in families.

Results : Using this approach, bioinformatic predictions showed that six mutations were found in our study: three mutations in the known genes of ZNF408, LRP5 and KIF11 and three mutations in the newly identified genes NR2E3,KRT3 and FOXL2 .To test the hypothesis that cases of FEVR are caused by NR2E3, KRT3 and FOXL2 , probands who were diagnosed as FEVR by a physician using wide-field fluorescein angiography were found to not have any mutations in any of the six known FEVR genes. Compared with previous reports, mutations in NR2E3, KRT3 and FOXL2 are believed to cause a broader spectrum of ocular disease. The NR2E3,KRT3 and FOXL2 genes likely play a role in retinal vascular development.

Conclusions: This report is the first to describe FEVR mutations in Yunnan province children with FEVR. This study would provide information on the genetic forms of the disease and direct counselling by analysing the genetic testing and genotype-phenotype interaction.

\section{Background}

Familial exudative vitreoretinopathy (FEVR, OMIM 133780) is a rare hereditary disorder characterized by anomalous retinal vascularization, including a retinal avascular zone, exudate, macular traction, retinal neovascularization, traction or rhegmatogenous retinal detachment[1,2] and affects the growth and development of blood vessels in the retina of the eye. FEVR is one of several paediatric vitreoretinopathies and can lead to visual impairment, including complete blindness in one or both eyes $[3,4]$. Although rare, FEVR is a potentially preventable cause of vision loss in patients younger than 30 years of age [5]. In 1969, Criswick and Schepens first described this disease [6], while in 1976, Canny and Oliver were the first to confirm the clinical features through the use of fundus fluorescein angiography [7]. Wide-field fluorescein angiography has become the gold standard from the diagnostic standpoint in the 
diagnosis and monitoring of FEVR [3]. However, FEVR has complicated and variable clinical manifestations, even between the individual eyes of a patient or among affected siblings in the same family, and its clinical manifestations differ greatly among patients [8-10]. Mild forms of FEVR can be asymptomatic and only exhibit peripheral retinal vascular abnormalities, such as venous telangiectasias, altered arterial tortuosity and a peripheral avascular zone [11]. Severe forms of FEVR differ greatly within the same family and are associated with subretinal and intraretinal haemorrhages, retinal neovascularization, retinal folds, exudates, and tractional retinal detachment $[3,12]$.

FEVR most often presents as visually significant in childhood, although it might progress at any age with sight-threatening manifestations [13]. In 1998, a 5-stage FEVR classification scheme was described by

Pendergast and Trese [14]. In Stage 1, the avascular periphery is notable, while Stage 2 occurs when neovascularization develops with the avascular periphery. Stage 3 is defined by macula-sparing retinal detachment and proceeds to Stage 4 with macula-involving retinal detachment. Stage 5 is complete retinal detachment, with the $A$ versus $B$ designations being for the absence versus the presence of exudate [13].

To date, 6 genes have been identified that account for some cases of FEVR. These genes are FZD4 (Frizzled 4 receptor), LRP5 (low-density-lipoprotein receptor-related protein 5), NDP(Norrie disease protein), TSPAN12 (tetraspanin-12), ZNF408 (zinc finger protein 408), and KIF11 (kinesin family member 11) [15-23]. Mutations in these gene have been implicated in abnormal signalling of known biological pathways and are inherited in autosomal dominant (AD), autosomal recessive (AR), and X-linked recessive fashions [3]. $A D$ is the most common mode of inheritance and is associated with mutations in FZD, LRP5 and TSPAN12. Mutations in LRP5 are associated with AR inheritance, whereas mutations in $N D P$ have caused $X$-linked recessive forms of the disease [24] $[25,26]$.

Though occurring almost 50 years ago, the first descriptions of FEVR were beautifully illustrated, and subsequently, the underlying pathophysiology, genetics, diagnosis, and management of the disease have been learned [3]. Only $40 \%$ to $50 \%$ of the patients with FEVR have had a corroborating genetic mutation identified, and the contribution of any given gene to the disease differs among study populations $[27,28]$.

However, the detailed mutational spectrum of these six genes was unknown in Chinese individuals [29]. Even genetic testing has not been carried out in Yunnan province, and there are few reports regarding mutations in the 25 ethnic minority groups in Yunnan. In the present study, genetic testing was performed on individuals with FEVR in Yunnan province to investigate the genetic causes of FEVR.

WES was used to sequence DNA from affected proband of FEVR pedigrees with no known causative FEVR gene mutations (FZD4, LRP5, NDP, TSPAN12, ZNF408, and KIF11). We identified three pedigrees

with a heterozygous ZNF408, LRP5 and KIF11 profile and three pedigrees with a heterozygous $N R 2 E 3, K R T 3$ and FOXL2 mutations. To test the hypothesis that more cases of FEVR are caused by 
NR2E3, KRT3 and FOXL2 mutations, we screened for mutations in the NR2E3, KRT3 and FOXL2 genes in a cohort of FEVR probands who did not have a mutation in any of the known FEVR genes.

\section{Results}

\section{Clinical findings}

\section{Patient 1}

The mutation, ZNF408, p.G290S, identified in a proband, was associated with a FEVR phenotype. The family, which has one girl (the proband) and unaffected parents, was recruited into the FEVR project specifically because of the fundus appearance mimicking FEVR. The proband was a 13-day-old baby at the time of recruitment.

\section{Patient 2}

The mutation in LRP5 involved in splicing, identified in a proband was associated with a stage 4-FEVR phenotype. The family, which has two girls (the proband, and an unaffected elder sister of proband) and unaffected parents, was recruited in the FEVR project specifically because of the stage 4- FEVR. The proband was a 3-year-old girl at the time of recruitment. She had been noted as have retinal abnormalities at age 3 years. She was born full-term with a birth weight of $3.2 \mathrm{~kg}$. The elder sister was examined at age 5 but she is normal.

\section{Patient 3}

The mutation in KIF11, p.1423T, identified in proband was associated with a FEVR phenotype. The family, which has two boys (the proband and an unaffected eld brother) and unaffected parents, was recruited into the FEVR project specifically because of stage-1FEVR. The proband was an 8-month-old baby at the time of recruitment. The retinal abnormalities were first noted at age 14 days. He was born full-term with a birth weight of $2.9 \mathrm{~kg}$. There was no family history of health problems compatible with a diagnosis of FEVR, and the mutation was found in his unaffected father and elder brother.

\section{Patient 4}

The mutations in NR2E3, p.H361R, identified in a proband were associated with a FEVR phenotype. The family, which has one boy (the proband) and an unaffected elder sister, as well as unaffected parents, was recruited into the FEVR project specifically because of stage-4 FEVR. The proband was a 3-year-old boy at the time of recruitment.

\section{Patient5}

The mutation, $K R T 3, \mathrm{p} . \mathrm{R} 271 \mathrm{H}$, identified in proband was associated with a FEVR phenotype. The family, which has one boy (the proband) and unaffected parents, was recruited in the FEVR project specifically because of stage-4FEVR. The proband was a 2-month-old boy at the time of recruitment. 


\section{Patient 6}

The FOXL2 mutation, p.333-337del, was identified in a proband was associated with a FEVR phenotype. The family, which has one boy (the proband) and unaffected parents, was recruited in the FEVR project specifically because of stage-4 FEVR. The proband was an 11-month-old boy at the time of recruitment.

\section{Genetic Analysis}

WES was initially performed on an affected probands (pedigree I- $\$ ) to identify a shared disease-causing variant. The reads aligned to the human genome, mapped to the target region with a mean coverage between 99.8 and 99.9\%, SNPs and indels are shown in Table 1. Between 11,631 and 11,844 were identified as SNPs from six affected individuals, whereas 833-918 indels were identified in the unaffected individuals. Analysis based on the pattern of inheritance identified common homozygous and heterozygous variants in six affected individuals (Table 1). Among the identified variants, the numbers of selected gene were displayed in Table 1 according to our mutational analysis. Further analysis based on expression profiles identified six candidate variants (Table 1). Among these, the four software packages predicted six of the missense variants to be damaging. After filtering for suspected sequencing artefacts and with a multiple allele frequency greater than $5 \%$, the shared missense or nonsense variants identified were displayed in Table 2.

Sanger sequencing revealed the novel, homozygous missense variants identified in these probands (Fig1 family tree, and Table 3); p.G290S in the ZNF408 gene, splicing in LRP5, p.I423T in KIF11, p.H361R in NR2E3, p.R271H in KRT3, p. 333_337del inFOXL2 segregating with disease in families 1,2,3,4,5,6, respectively (Fig. 2).

In the families (pedigree I- $\mathrm{X}$ ), the remaining heterozygous, ZNF408, LRP5 and KIF11 genes were selected as the candidates because heterozygous mutations in these genes has previously been reported as being

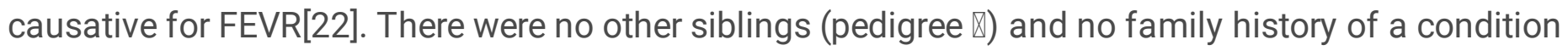
compatible with a diagnosis of FEVR. The mutation was absent father, mother, and mother in family I,, ,

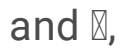

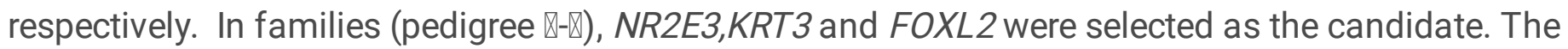

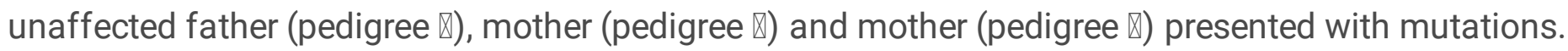

Sanger sequencing identified additional novel heterozygous mutations in 6 probands (Figure 1 family tree).

Gene type, amino acid changes and nucleotide changes of all mutations are shown in Table 3 , and the amino acid changes in family2 (LRP5) are in an acceptor splice site.

Additionally, these variants were absent in 60 normal control individuals.

\section{Discussion}


In this study, a comprehensive molecular screen for mutations in six families with FEVR was performed with a WES approach. In these six families, the probands were found by fluorescein angiography, which is critical for the diagnosis and management in FEVR [30]. Several bioinformatics tools predicted a strong effect on the amino acid changes inducing exons 5, 6, 11, 8, 25 and 18, in families 1, 2, 3, 4, 5 and 6 , respectively. These novel variants found in our patients were neither reported in the literature nor in public databases. To investigate the effects of mutation on the transcript, we analysed ZNF408, LRP5, KIF11, NR2E, KRT3 and FOXL2 DNA obtained from patient's whole blood. The potential regions were amplified by standard PCR. The nucleotide substitutions in these families caused the amino acid changes in exons. As a result, we were able to obtain accurate mutations in FEVR disease gene from these families (pedigree I-III) and identify putative pathogenic mutations for $50 \%$ of the patients from genes that are published in the literature. We were also able to find mutation in three genes, NR2E3, KRT3 and FOXL2, in the families (pedigree III-VI).

In these three families (pedigree I-III), there were newly occurring mutations, with most of the alleles identified having never been reported on before. Through previously reported work, it is known that a number of variants discovered in the genes ZNF408, LRP5 and KIF11 correlate with FEVR. Our study allowed us to obtain an accurate estimation of the number of patients carrying mutations in each FEVR gene. In our patient cohort, ZNF408, LRP5, and KIF11, which are the most frequently mutated, were found in families (pedigree I-III). In one Dutch family and a Japanese family, novel mutations in ZNF408 have been reported. Subsequently, it has been shown to play a putative role in retinal vasculogenesis through the use of a knockdown of znf408 in zebrafish [31]. That mutations in LRP5 can lead to abnormal retinal vascular formation, delayed retinal vascular development and subsequent pathological glomeruloid vessels was demonstrated by the use of a $L r p 5$ knockout ( $L r p 5-/-)$ mouse model of FEVR [32]. The $L R P 5$ gene has been shown to exhibit two inheritance patterns, $A D$ and $A R$. Due to compound heterozygous genotypes consisting of missense mutations, AR of $L R P 5$ is most common [33]. AR inheritance patterns have been observed in our study. This proband carries compound heterozygous mutation, and LRP5 mutations are found in the unaffected father, while only wild-type variants are present in the unaffected mother. Mutations in KIF11 are associated with microcephaly, chorioretinopathy, or mental retardation [34]. Mutations of this gene have been recently identified in individuals affected with FEVR. Furthermore, there is a phenotypic overlap between the two diseases caused by KIF11 mutations [35-37]. With this hypothesis, the mutations of NR2E3, KRT3 and FOXL2 had been found in three patients with FEVR. In 2000, the nuclear receptor subfamily 2, group E, member 3 gene (NR2E3) on chromosome 15q23, which encodes a ligand-dependent transcription factor, was found to play an important role in photoreceptor development and differentiation and is responsible for the enhanced S-cone syndrome (ESCS) and was identified [38, 39]. Subsequently, mutations in NR2E3 were found in patients with Goldmann-Favre syndrome and clumped pigmentary retinal degeneration [40], autosomal recessive retinitis pigmentosa, and dominant retinitis pigmentosa [41]. These mutations of NR2E3 gene expanded the phenotypic variations for people with retinal dystrophy. Keratins are a group of structural proteins in the epithelia of cornea [42]. In 1997, keratin 3 (KRT3) was first linked to mutations in Meesmann's corneal dystrophy [43].The gene family of Fork head box (Fox), which named after 
the Drosophila melanogaster fork head gene $(f k h)$, is an evolutionarily ancient gene family[44]. The Forkhead Box L2 gene (FOXL2) which encodes the fork head transcription factor FOXL2 and plays an important role in thedevelopment of the eyelids and ovary, is the primary gene that underlies

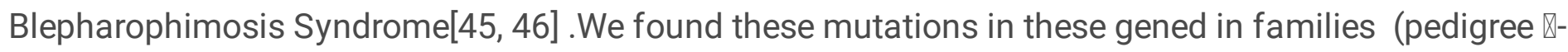
区), with FEVR. Further research needs to be carried out on these genes: NR2E, KRT3 and FOXL2.

To the best of our knowledge, the roles of NR2E3, KRT3 and FOXL2 were first reported in this study, and the expression patterns of $N R 2 E 3, K R T 3$ and FOXL2 in different cell types are still not fully defined. Interestingly, traces of $N R 2 E 3, K R T 3$ and FOXL2 transcript in whole blood samples were found to allow for the direct study of the effects of the mutations, making the in vitro model redundant for the mutation assay. Further research will clarify the roles of the NR2E3, KRT3 and FOXL2 genes in the development of retinal vessels.

\section{Conclusion}

In summary, the use of the WES method in this study allows us to efficiently identify all of the most likely causative variants in disease-associated genes associated with FEVR.. Mutations in these genes cause a broader spectrum of ocular disease than previously reported. The molecular diagnosis together with the clinical phenotype provides potentially useful information for the diagnosis of the disease, the prognosis for the patient, and guidance for genetic counseling. This information is particularly valuable, given the phenotypic variation found even within the same family.

\section{Methods}

\section{Participants and Clinical Data Collection}

This study was approved by the Ethics Committee of the Children's Hospital of Kunming Medical University, and written informed consent was obtained from either the participants or their guardians. No stipend was offered.

With the aim of identifying FEVR genes and describing the phenotypic spectrum of the disease, participants were recruited from six nonconsanguineous families by the Children's Hospital of Kunming Medical University (Kunming, China). In these families, children suffered from FEVR; however, the parents and other members of the family were normal. Additionally, our study enrolled 60 individuals of normal control aged between 6 and 35 years old, including 35 males and 25 females without associated hereditary diseases.

Clinical data were collected prospectively by the referring physician. These data included results from eye examinations and, whenever possible, ultrasonography and fundus photography. Parents and siblings at risk were also invited to participate and an eye examination was performed, including best-corrected 
visual acuity, ocular alignment, slit lamp examination, dilated fundus examination and, in some cases, intravenous fluorescein angiography (IVFA).

The ethnicity of each participant was recorded by the referring physician who completed the study history questionnaire. This information was used in the analysis of the novel mutation screening.

\section{WES and variant analysis}

According to the principles of the Declaration of Helsinki, two-millilitre peripheral blood samples were collected from the probands, their parents, and siblings in tubes containing 0.2 M EDTA. Genomic DNA extraction was performed on these samples using standard protocols. Briefly, DNA was extracted from the venous blood of each subject with the QIAamp DNA blood extraction kit (TIANGEN, Beijing, China). Following extraction, 3 micrograms of genomic DNA was fragmented by Covaris 32, and the 3 ' end of each DNA fragment was A-tailed according to the manufacturer's protocol (MyGenostics, Inc., Beijing, China), Then, Illumina adapters were ligated to these fragments. To obtain a 350-400 base-pair product, all samples were checked with Nanodrop 2000 or Qubit systems to determine if they represented a captured library.

Each qualified captured library was loaded on an Illumina MiSeq 2000 sequencing platform, and the sequences were determined to ensure that each sample met the desired average sequencing coverage.

\section{Mutation analysis}

Raw image files were processed for base calling and raw data generation with Bcl2Fastq software (Bcl2Fastq 2.18.0.12, Illumina, Inc.). In addition, low-quality variations were filtered out to achieve a quality score $\geq 20$. Then, the clean and high-quality reads were aligned to the reference human genome (UCSC hg19, http://genome.ucsc.edu/) using Short Oligonucleotide Analysis Package (SOAP) aligner software (SOAP2.21, soap.genomics.org.cn/soapsnp.html).

The polymerase chain reaction (PCR) duplicates were removed by using the Picard programme [47] [48]. The single nucleotide polymorphisms (SNPs) were determined by using the SOAP programme[49]. The reads were realigned using Burrows-Wheeler Aligner (BWA) software 0.7.15, and the deletions and insertions (indels) were detected with Genome Analysis Toolkit software 3.7. In addition, the identified indel SNPs were annotated with the Exome-assistant programme (http://122.228.158.106/exomeassistant). The non-synonymous variants were evaluated to determine their pathogenicity by four algorithms, namely, PolyPhen (http://genetics.bwh.harvard.edu/pph2/), Protein Analysis Through Evolutionary Relationships (PANTHER, www.pantherdb.org), Sorting Intolerant from Tolerant (SIFT, http://sift.jcvi.org/) and Pathogenic Mutation Prediction (Pmut, http://mmb.pcb.ub.es/PMut/). 


\section{Mutation validation}

In these families, Sanger sequencing and PCR (polymerase chain reaction) with an ABI3500 sequencer were used to confirm potential causative variants. Regions sequenced included the 5 coding exons of the ZNF408 gene, the 6 coding exons of the LRP5 gene, the 11 exons of the KIF11 gene (including the $5^{\prime}$ untranslated and promoter regions), the 8 coding exons of the NR2E3 gene, the 2 coding exons of the KRT3 gene, the 1coding exons of the FOXL2 gene. The sites of variation were identified in order to compare the DNA sequences using the corresponding GenBank (www.ncbi.nIm.nih.gov) reference sequences. Table 1 displays the sequences of forward and reverse primers. Thermocycling conditions were as follows: an initial denaturation of $95^{\circ} \mathrm{C}$ for $10 \mathrm{~min}, 35$ cycles of denaturation at $94^{\circ} \mathrm{C}$ for $30 \mathrm{sec}$., annealing at $64^{\circ} \mathrm{C}$ for $30 \mathrm{sec}$., extension at $72^{\circ} \mathrm{C}$ for $45 \mathrm{sec}$. and a final extension of $72^{\circ} \mathrm{C}$ for $5 \mathrm{~min}$. The sequences of forward and reverse primers are in Table 4.

\section{Availability Of Data And Materials}

The data sets used and analysed during this study are available from the corresponding author on reasonable request.

\section{Abbreviations}

FEVR: Familial exudative vitreoretinopathy

SNVs: single nucleotide variants

InDels: small insertions/deletions

FZD4: Frizzled 4 receptor

LRP5: low-density-lipoprotein receptor-related protein 5

NDP: Norrie disease protein

TSPAN12: tetraspanin-12

ZNF408: zinc finger protein 408

KIF11: kinesin family member 11

AD: autosomal dominant

AR: autosomal recessive

NR2E3: nuclear receptor subfamily 2, group E, member 3 gene 
KRT3: keratin 3

FOXL2: Forkhead Box L2 gene

IVFA: intravenous fluorescein angiography

SOAP: Short Oligonucleotide Analysis Package

PCR: polymerase chain reaction

SNPs: single nucleotide polymorphisms

SIFT: Sorting Intolerant from Tolerant

\section{Declarations}

\section{Acknowledgments}

We thank all subjects who participated in this study.

\section{Funding}

This work was supported by a grant from the Health and Family Planning Commission of Kunming [grants nos. 2017-SW(Yang)-02], Key R \& D plan of Yunnan Provincial Department of science and technology--International cooperation in science and technology (grants nos. 2018IA047) and the Joint Fund of the Yunnan Science and Technology Department and Kunming Medical-University [grants nos. 2019FE001(-274)] respectively.

\section{Author information}

\section{Author notes}

Zhen Zhang, Yi-shuang Xiao, Hong-chao Jiang should be considered joint first author.

\section{Contributions}

Zhen Zhang, Yi-shuang Xiao and Hong-chao Jiang conceived and designed the experiments; Zhen Zhang, Ru Shen and Jing Ma performed the experiments;Zhen Zhang, Yi-shuang Xiao, Jing Ma, Hong-chao Jiang, Xiao-hong Yang and LiTang analysed the data; Yi-shuang Xiao,recruited patients and collected clinical information. Yi-shuang Xiao and Huai-yu Gu contributed to accumulation and interpretation of clinical data. Wen-Ji He and Zhen Zhang coordinated the project.All authors read and approved the final manuscript. 
Correspondence to Wen-Ji He and Jing Ma.

\section{Ethics approval and consent to participate}

This study was approved by the ethics committee of the Children's Hospital of Kunming Medical University. All the patients and their related family members accepting the genetic testing assigned a consent form.

\section{Consent for publication}

The authors give their consent for publication of this manuscript.

Competing interests

The authors declare that they have no competing interests.

\section{References}

1. Shastry BS, Trese MT: Cosegregation of two unlinked mutant alleles in some cases of autosomal dominant familial exudative vitreoretinopathy. EUR J HUM GENET 2004, 12(1):79-82.

2. Kashani AH, Brown KT, Chang E, Drenser KA, Capone A, Trese MT: Diversity of retinal vascular anomalies in patients with familial exudative vitreoretinopathy. OPHTHALMOLOGY 2014, 121(11):2220-2227.

3. Tauqeer Z, Yonekawa Y: Familial Exudative Vitreoretinopathy: Pathophysiology, Diagnosis, and Management. Asia Pac J Ophthalmol (Phila) 2018, 7(3):176-182.

4. Shastry BS: Genetic susceptibility to advanced retinopathy of prematurity (ROP). J BIOMED SCI 2010, 17:69.

5. Quiram PA, Drenser KA, Lai MM, Capone AJ, Trese MT: Treatment of vascularly active familial exudative vitreoretinopathy with pegaptanib sodium (Macugen). Retina 2008, 28(3 Suppl):S8-S12.

6. Criswick VG, Schepens CL: Familial exudative vitreoretinopathy. AM J OPHTHALMOL 1969, 68(4):578-594.

7. Canny $\mathrm{CL}$, Oliver GL: Fluorescein angiographic findings in familial exudative vitreoretinopathy. Arch Ophthalmol 1976, 94(7):1114-1120. 
8. Ober RR, Bird AC, Hamilton AM, Sehmi K: Autosomal dominant exudative vitreoretinopathy. $\mathrm{Br} J$ Ophthalmol 1980, 64(2):112-120.

9. Pendergast SD, Trese MT: Familial exudative vitreoretinopathy. Results of surgical management. OPHTHALMOLOGY 1998, 105(6):1015-1023.

10. van Nouhuys CE: Signs, complications, and platelet aggregation in familial exudative vitreoretinopathy. AM J OPHTHALMOL 1991, 111(1):34-41.

11. Li JK, Fei P, Li Y, Huang QJ, Zhang Q, Zhang X, Rao YQ, Li J, Zhao P: Identification of novel KIF11 mutations in patients with familial exudative vitreoretinopathy and a phenotypic analysis. Sci Rep 2016, 6:26564.

12. Gilmour DF: Familial exudative vitreoretinopathy and related retinopathies. Eye (Lond) 2015, 29(1):114.

13. Lee J, El-Dairi MA, Tran-Viet D, Mangalesh S, Dandridge A, Jiramongkolchai K, Viehland C, Toth CA: LONGITUDINAL CHANGES IN THE OPTIC NERVE HEAD AND RETINA OVER TIME IN VERY YOUNG CHILDREN WITH FAMILIAL EXUDATIVE VITREORETINOPATHY. Retina 2017.

14. Pendergast SD, Trese MT: Familial exudative vitreoretinopathy. Results of surgical management. OPHTHALMOLOGY 1998, 105(6):1015-1023.

15. Robitaille J, MacDonald ML, Kaykas A, Sheldahl LC, Zeisler J, Dube MP, Zhang LH, Singaraja RR, Guernsey DL, Zheng B et al: Mutant frizzled-4 disrupts retinal angiogenesis in familial exudative vitreoretinopathy. NAT GENET 2002, 32(2):326-330.

16. Chen ZY, Battinelli EM, Fielder A, Bundey S, Sims K, Breakefield XO, Craig IW: A mutation in the Norrie disease gene (NDP) associated with X-linked familial exudative vitreoretinopathy. NAT GENET 1993, 5(2):180-183.

17. Toomes C, Bottomley HM, Jackson RM, Towns KV, Scott S, Mackey DA, Craig JE, Jiang L, Yang Z, Trembath R et al: Mutations in LRP5 or FZD4 underlie the common familial exudative vitreoretinopathy locus on chromosome 11q. AM J HUM GENET 2004, 74(4):721-730.

18. Jiao X, Ventruto V, Trese MT, Shastry BS, Hejtmancik JF: Autosomal recessive familial exudative vitreoretinopathy is associated with mutations in LRP5. AM J HUM GENET 2004, 75(5):878-884.

19. Poulter JA, Ali M, Gilmour DF, Rice A, Kondo H, Hayashi K, Mackey DA, Kearns LS, Ruddle JB, Craig JE et al: Mutations in TSPAN12 cause autosomal-dominant familial exudative vitreoretinopathy. $A M \mathrm{~J}$ HUM GENET 2010, 86(2):248-253.

20. Nikopoulos K, Gilissen C, Hoischen A, van Nouhuys CE, Boonstra FN, Blokland EA, Arts P, Wieskamp $\mathrm{N}$, Strom TM, Ayuso $\mathrm{C}$ et al: Next-generation sequencing of a $40 \mathrm{Mb}$ linkage interval reveals TSPAN12 mutations in patients with familial exudative vitreoretinopathy. AM J HUM GENET 2010, 86(2):240247.

21. Collin RW, Nikopoulos K, Dona M, Gilissen C, Hoischen A, Boonstra FN, Poulter JA, Kondo H, Berger W, Toomes $C$ et al: ZNF408 is mutated in familial exudative vitreoretinopathy and is crucial for the development of zebrafish retinal vasculature. Proc Natl Acad Sci U S A 2013, 110(24):9856-9861. 
22. Robitaille JM, Gillett RM, LeBlanc MA, Gaston D, Nightingale M, Mackley MP, Parkash S, Hathaway J, Thomas A, Ells A et al: Phenotypic overlap between familial exudative vitreoretinopathy and microcephaly, lymphedema, and chorioretinal dysplasia caused by KIF11 mutations. JAMA OPHTHALMOL 2014, 132(12):1393-1399.

23. Hu H, Xiao X, Li S, Jia X, Guo X, Zhang Q: KIF11 mutations are a common cause of autosomal dominant familial exudative vitreoretinopathy. Br J Ophthalmol 2016, 100(2):278-283.

24. Toomes C, Bottomley HM, Jackson RM, Towns KV, Scott S, Mackey DA, Craig JE, Jiang L, Yang Z, Trembath R et al: Mutations in LRP5 or FZD4 underlie the common familial exudative vitreoretinopathy locus on chromosome 11q. AM J HUM GENET 2004, 74(4):721-730.

25. Robitaille JM, Wallace K, Zheng B, Beis MJ, Samuels M, Hoskin-Mott A, Guernsey DL: Phenotypic overlap of familial exudative vitreoretinopathy (FEVR) with persistent fetal vasculature (PFV) caused by FZD4 mutations in two distinct pedigrees. OPHTHALMIC GENET 2009, 30(1):23-30.

26. Poulter JA, Ali M, Gilmour DF, Rice A, Kondo H, Hayashi K, Mackey DA, Kearns LS, Ruddle JB, Craig JE et al: Mutations in TSPAN12 cause autosomal-dominant familial exudative vitreoretinopathy. $A M J$ HUM GENET 2010, 86(2):248-253.

27. Seo SH, Yu YS, Park SW, Kim JH, Kim HK, Cho SI, Park H, Lee SJ, Seong MW, Park SS et al: Molecular Characterization of FZD4, LRP5, and TSPAN12 in Familial Exudative Vitreoretinopathy. Invest Ophthalmol Vis Sci 2015, 56(9):5143-5151.

28. Salvo J, Lyubasyuk V, Xu M, Wang H, Wang F, Nguyen D, Wang K, Luo H, Wen C, Shi C et al: Nextgeneration sequencing and novel variant determination in a cohort of 92 familial exudative vitreoretinopathy patients. Invest Ophthalmol Vis Sci 2015, 56(3):1937-1946.

29. Rao FQ, Cai XB, Cheng FF, Cheng W, Fang XL, Li N, Huang XF, Li LH, Jin ZB: Mutations in LRP5,FZD4, TSPAN12, NDP, ZNF408, or KIF11 Genes Account for 38.7\% of Chinese Patients With Familial Exudative Vitreoretinopathy. Invest Ophthalmol Vis Sci 2017, 58(5):2623-2629.

30. Kashani AH, Brown KT, Chang E, Drenser KA, Capone A, Trese MT: Diversity of retinal vascular anomalies in patients with familial exudative vitreoretinopathy. OPHTHALMOLOGY 2014, 121(11):2220-2227.

31. Collin RW, Nikopoulos K, Dona M, Gilissen C, Hoischen A, Boonstra FN, Poulter JA, Kondo H, Berger W, Toomes $\mathrm{C}$ et al: ZNF408 is mutated in familial exudative vitreoretinopathy and is crucial for the development of zebrafish retinal vasculature. Proc Natl Acad Sci U S A 2013, 110(24):9856-9861.

32. Wang Z, Liu CH, Sun Y, Gong Y, Favazza TL, Morss PC, Saba NJ, Fredrick TW, He X, Akula JD et al: Pharmacologic Activation of Wnt Signaling by Lithium Normalizes Retinal Vasculature in a Murine Model of Familial Exudative Vitreoretinopathy. AM J PATHOL 2016, 186(10):2588-2600.

33. Salvo J, Lyubasyuk V, Xu M, Wang H, Wang F, Nguyen D, Wang K, Luo H, Wen C, Shi C et al: Nextgeneration sequencing and novel variant determination in a cohort of 92 familial exudative vitreoretinopathy patients. Invest Ophthalmol Vis Sci 2015, 56(3):1937-1946.

34. Ostergaard P, Simpson MA, Mendola A, Vasudevan P, Connell FC, van Impel A, Moore AT, Loeys BL, Ghalamkarpour A, Onoufriadis A et al: Mutations in KIF11 cause autosomal-dominant microcephaly 
variably associated with congenital lymphedema and chorioretinopathy. AM J HUM GENET 2012, 90(2):356-362.

35. Robitaille JM, Gillett RM, LeBlanc MA, Gaston D, Nightingale M, Mackley MP, Parkash S, Hathaway J, Thomas A, Ells A et al: Phenotypic overlap between familial exudative vitreoretinopathy and microcephaly, lymphedema, and chorioretinal dysplasia caused by KIF11 mutations. JAMA OPHTHALMOL 2014, 132(12):1393-1399.

36. Hu H, Xiao X, Li S, Jia X, Guo X, Zhang Q: KIF11 mutations are a common cause of autosomal dominant familial exudative vitreoretinopathy. Br J Ophthalmol 2016, 100(2):278-283.

37. Li JK, Fei P, Li Y, Huang QJ, Zhang Q, Zhang X, Rao YQ, Li J, Zhao P: Identification of novel KIF11 mutations in patients with familial exudative vitreoretinopathy and a phenotypic analysis. Sci Rep 2016, 6:26564.

38. Haider NB, Jacobson SG, Cideciyan AV, Swiderski R, Streb LM, Searby C, Beck G, Hockey R, Hanna DB, Gorman S et al: Mutation of a nuclear receptor gene, NR2E3, causes enhanced S cone syndrome, a disorder of retinal cell fate. NAT GENET 2000, 24(2):127-131.

39. Kobayashi M, Takezawa S, Hara K, Yu RT, Umesono Y, Agata K, Taniwaki M, Yasuda K, Umesono K: Identification of a photoreceptor cell-specific nuclear receptor. Proc Natl Acad Sci U S A 1999, 96(9):4814-4819.

40. Sharon D, Sandberg MA, Caruso RC, Berson EL, Dryja TP: Shared mutations in NR2E3 in enhanced Scone syndrome, Goldmann-Favre syndrome, and many cases of clumped pigmentary retinal degeneration. Arch Ophthalmol 2003, 121(9):1316-1323.

41. Coppieters F, Leroy BP, Beysen D, Hellemans J, De Bosscher K, Haegeman G, Robberecht K, Wuyts W, Coucke PJ, De Baere E: Recurrent mutation in the first zinc finger of the orphan nuclear receptor NR2E3 causes autosomal dominant retinitis pigmentosa. AM J HUM GENET 2007, 81(1):147-157.

42. Chen JL, Lin BR, Gee KM, Gee JA, Chung DD, Frausto RF, Deng SX, Aldave AJ: Identification of presumed pathogenic KRT3 and KRT12 gene mutations associated with Meesmann corneal dystrophy. MOL VIS 2015, 21:1378.

43. Irvine AD, Corden LD, Swensson O, Swensson B, Moore JE, Frazer DG, Smith FJ, Knowlton RG, Christophers E, Rochels R et al: Mutations in cornea-specific keratin K3 or K12 genes cause Meesmann's corneal dystrophy. NAT GENET 1997, 16(2):184-187.

44. Kwak HJ, Ryu KB, Medina JB, Park SC, Cho SJ: Temporal and spatial expression of the Fox gene family in the Leech Helobdella austinensis. J Exp Zool B Mol Dev Evol 2018.

45. Cocquet J, Pailhoux E, Jaubert F, Servel N, Xia X, Pannetier M, De Baere E, Messiaen L, Cotinot C, Fellous $\mathrm{M}$ et al: Evolution and expression of FOXL2. J MED GENET 2002, 39(12):916-921.

46. Crisponi L, Deiana M, Loi A, Chiappe F, Uda M, Amati P, Bisceglia L, Zelante L, Nagaraja R, Porcu S et al: The putative forkhead transcription factor FOXL2 is mutated in blepharophimosis/ptosis/epicanthus inversus syndrome. NAT GENET 2001, 27(2):159-166.

47. Etherington GJ, Ramirez-Gonzalez RH, MacLean D: bio-samtools 2: a package for analysis and visualization of sequence and alignment data with SAMtools in Ruby. BIOINFORMATICS 2015, 
31(15):2565-2567.

48. Li H, Handsaker B, Wysoker A, Fennell T, Ruan J, Homer N, Marth G, Abecasis G, Durbin R: The Sequence Alignment/Map format and SAMtools. BIOINFORMATICS 2009, 25(16):2078-2079.

49. Li R, Yu C, Li Y, Lam TW, Yiu SM, Kristiansen K, Wang J: SOAP2: an improved ultrafast tool for short read alignment. BIOINFORMATICS 2009, 25(15):1966-1967.

\section{Tables}

Table 1 Part of Data of Analysis

\begin{tabular}{|c|c|c|c|c|c|c|c|c|c|}
\hline \multirow[t]{2}{*}{ No. } & \multirow{2}{*}{$\begin{array}{c}\text { Reads } \\
\text { (M } \square\end{array}$} & \multirow{2}{*}{$\begin{array}{c}\text { Read } \\
\text { length } \\
\text { (bp) }\end{array}$} & \multirow{2}{*}{$\begin{array}{l}\text { Reads aligned to the } \\
\text { human genome }\end{array}$} & \multirow{2}{*}{$\begin{array}{l}\text { Reads mapped to the } \\
\text { target region }\end{array}$} & \multirow{2}{*}{$\begin{array}{c}\text { Mean } \\
\text { coverage }\end{array}$} & \multicolumn{2}{|c|}{ SNPs } & \multicolumn{2}{|c|}{ Indels, } \\
\hline & & & & & & $\begin{array}{c}\text { Non- } \\
\text { synonymous }\end{array}$ & $\begin{array}{c}\text { Splice } \\
\text { sites }\end{array}$ & $\begin{array}{c}\text { Coding } \\
\text { sequence }\end{array}$ & $\begin{array}{c}\text { Splice } \\
\text { sites }\end{array}$ \\
\hline 1 & 68.4 & 148 & 10155.88 & 10196.76 & $99.92 \%$ & 10959 & 773 & 622 & 296 \\
\hline 2 & 92.0 & 149 & 13700.82 & 13752.12 & $99.87 \%$ & 10887 & 828 & 598 & 235 \\
\hline 3 & 96.1 & 149 & 14283.23 & 14337.44 & $99.88 \%$ & 11027 & 817 & 625 & 249 \\
\hline 4 & 74.6 & 149 & 11098.47 & 11137.29 & $99.86 \%$ & 10840 & 791 & 660 & 230 \\
\hline 5 & 113.5 & 148 & 16803.20 & 16881.81 & $99.84 \%$ & 10980 & 816 & 628 & 249 \\
\hline 6 & 113.2 & 148 & 16729.23 & 16790.3 & $99.87 \%$ & 10983 & 826 & 630 & 236 \\
\hline
\end{tabular}

Table 2 The filtering for suspected sequencing artifacts

\begin{tabular}{|c|c|c|c|c|c|c|c|}
\hline No. & Unknown & Synonymous & Nonsynonymous & Stopgain & Splicing & Nonframeshift & Frameshift \\
\hline 1 & 11 & - & 259 & 5 & 20 & 15 & 10 \\
\hline 2 & 17 & - & 355 & 13 & 19 & 20 & 29 \\
\hline 3 & 5 & - & 265 & 4 & 20 & 8 & 9 \\
\hline 4 & 4 & 232 & 241 & 4 & 15 & 17 & 8 \\
\hline 5 & 9 & - & 253 & 5 & 20 & 25 & 11 \\
\hline 6 & 9 & 2 & 226 & 5 & 19 & 15 & 6 \\
\hline
\end{tabular}

Table 3 Gene mutations of family $1-6$.

\begin{tabular}{|c|c|c|c|c|c|c|}
\hline Gene & Exon & Amino Acid Changes & ID & Nucleotide Changes & Gene Type & Inheritance mode \\
\hline ZNF408 & 5 & p.G290S & chr11-46726142 & c.868G $>$ A & het & AD \\
\hline LRP5 & 6 & splicing & chr11-68153782 & c.1016-2A $>$ T & het & AR \\
\hline KIF11 & 11 & p.I423T & chr10-94388615 & c.1268T $>$ C & het & AD \\
\hline NR2E3 & 8 & p.H361R & chr15-72106440 & c.1082A $>$ G & het & AR \\
\hline KRT3 & 2 & p.R271H & chr12-53187949 & c.812G $>$ A & het & AD \\
\hline FOXL2 & 1 & p.333_337del & chr3-138664553-138664568 & c.997_1011del & het & AD \\
\hline
\end{tabular}




\begin{tabular}{|c|c|c|c|}
\hline Gene & Exon & Forward primers & Reverse primers \\
\hline ZNF408 & 5 & TAAGTTCCCAACCCAGGACC & TGTACCACCTGGTGCTCTTTG \\
\hline LRP5 & 6 & ACCAGCCTTTGCAAGGAGAG & CGTGTCGGTCCAGTAGAGGT \\
\hline KIF11 & 11 & ATTGAGGGCAAGGGAAAAAT & GATCACGCTATTGCACTCCA \\
\hline$N R 2 E 3$ & 8 & GCCACTCCTGGTTGACTGTG & TGAACTGAGACCCTTGTGCTG \\
\hline KRT3 & 2 & TTGCTGGAATTGAGGATTGTG & TGAATACTTCCATTCATGCCC \\
\hline FOXL2 & 1 & TACCTGCAGTCTGGCTTCCT & CAGAGGGTGTGAGGTCAGG \\
\hline
\end{tabular}

\section{Figures}

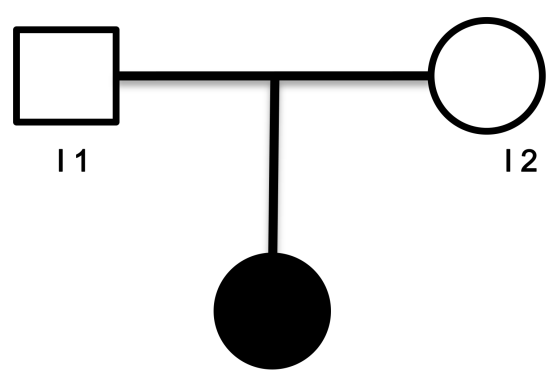

II1

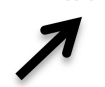

Family 1

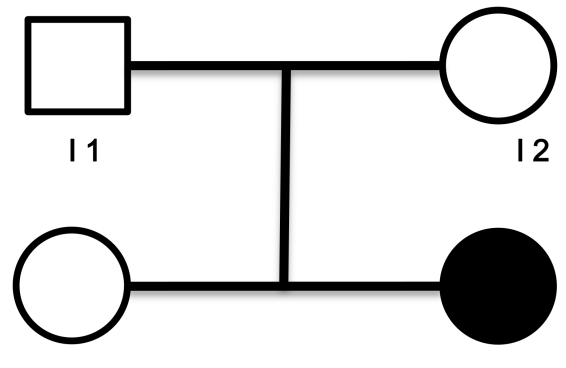

II1

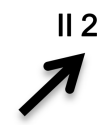

Family 2

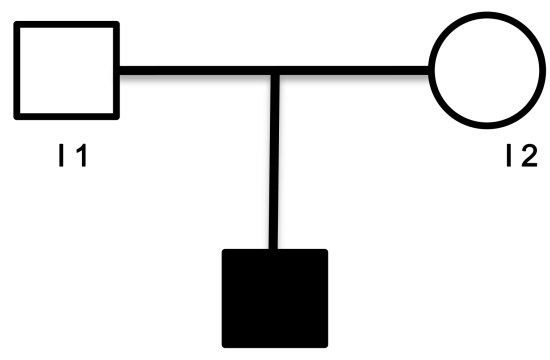

II1

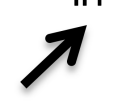

Family 5

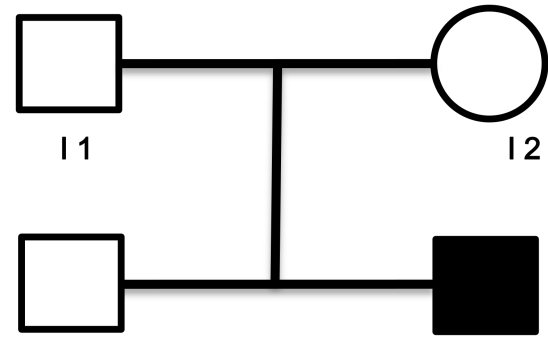

II1

Family 3

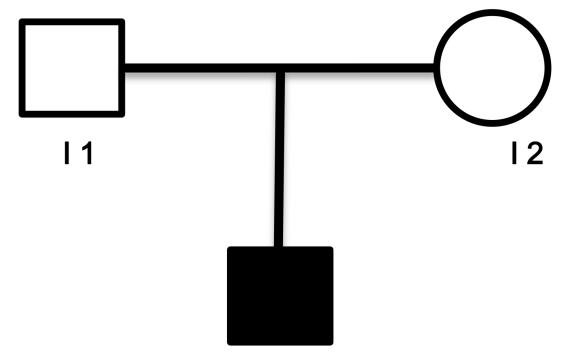

II1

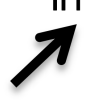

Family 6

Figure 1

Pedigrees of family with FEVR. Unaffected subjects are denoted as blank while affected subjects are represented with darkened symbols. The arrow indicates the proband. In all families, I 1 are the 
unaffected father and I 2 are the unaffected mother; In families 1, 5 and 6, II 1 are the proband; In families 2, 3 and 4, II 1 are the unaffected sibling while II 2 are the proband.

II 1

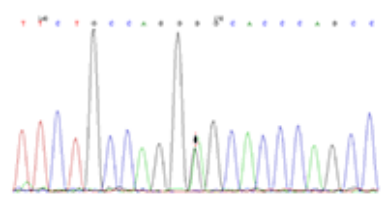

I1

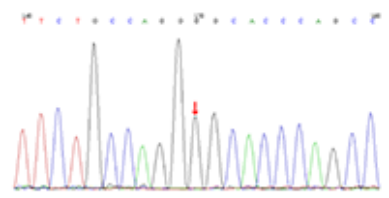

12

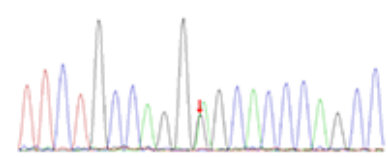

A

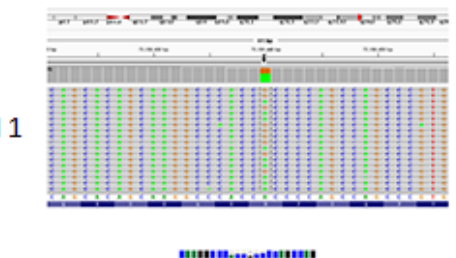

II 2
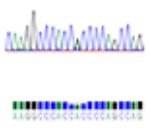

I1

12

II 1

11

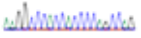

I1

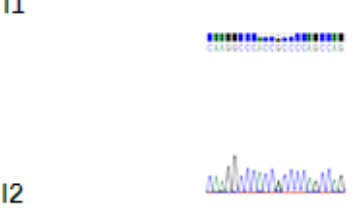

D
I1

anmonamanom

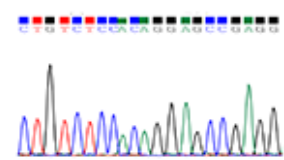

B
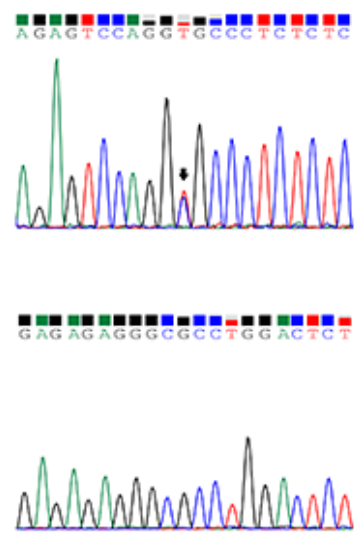

II 1
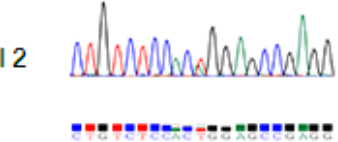

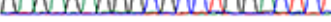

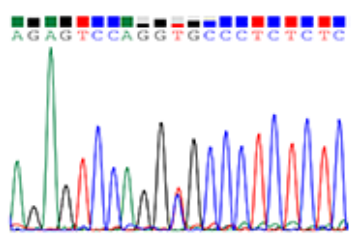

I2

E
II 1

Lhollowidennems

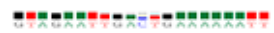

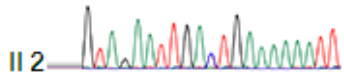

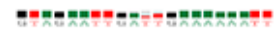

I1

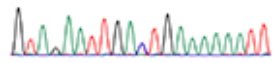

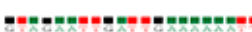

12

Anrowarenclumans

C

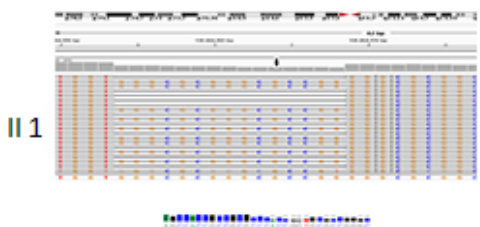

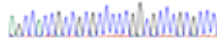

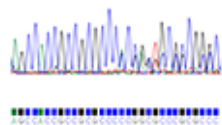

F

\section{Figure 2}

Partial electropherograms of the genomic region covering the genes: (A) The heterozygous ZNF408 c.868G >A variant of the proband in family 1 ; (B) The heterozygous LRP5 c.1016-2A>T variant of the proband in family 2; (C) The heterozygous KIF11 c.1268T>C variant of the proband in family 3; (D) The heterozygous NR2E3 c.1082A $>\mathrm{G}$ variant of the proband in family 4; $(\mathrm{E})$ The heterozygous KRT3 c.812G>A 
variant of the proband in family 5; (F) The heterozygous FOXL2 c.997_1011del variant of the proband in family 6.Arrows denote the mutations. II 1 the proband; II 2 unaffected sibling; I 1 the unaffected father; I 2 the unaffected mother 